Running Head: IMPLEMENTATION INTENTIONS AND EFFORT

\title{
Goal Striving Strategies and Effort Mobilization: When Implementation Intentions Reduce Effort-related Cardiac Activity during Task Performance
}

\author{
Laure Freydefont $^{1} \quad$ Peter M. Gollwitzer $^{12} \quad$ Gabriele Oettingen $^{13}$ \\ ${ }^{1}$ New York University \\ ${ }^{2}$ University of Konstanz \\ ${ }^{3}$ University of Hamburg
}

Author note

Laure Freydefont, Psychology Department, New York University, New York, NY, USA;

Peter M. Gollwitzer, Psychology Department, New York University, New York, NY, USA, and Psychology Department, University of Konstanz, Konstanz, Germany; Gabriele Oettingen, Psychology Department, New York University, New York, NY, USA, and Psychology Department, University of Hamburg, Hamburg, Germany.

Correspondence concerning this article should be addressed to Laure Freydefont, Motivation Lab, New York University, Psychology Department, 4 Washington Place, 10003 New York, NY, USA. E-mail: laure.freydefont@nyu.edu

Keywords: goal striving strategies, implementation intentions, goal intentions, effort mobilization, self-regulation 


\section{Goal Striving Strategies and Effort Mobilization: When Implementation Intentions Reduce Effort-Related Cardiac Activity during Task Performance}

Success in goal attainment is highly increased by forming plans. While goal intentions are built as "I intend to achieve X!" with $X$ representing a desired future, outcome or behavior, implementation intentions are plans with the format of "If I encounter situation X, then I will initiate response Y!" Forming an if-then plan is a self-regulatory goal-striving strategy facilitating goal attainment (Gollwitzer, 1993, 1999, 2014). Such plans link an anticipated critical situation (i.e., opportunity or obstacle) to an instrumental goal-directed cognitive, affective, or behavioral response. While goal intentions specify a desired future only, implementation intentions spell out when, where (by specifying the if-component) and how (by specifying the then-component) this desired future shall be attained. It has been demonstrated in extensive research that adding implementation intentions to one's goal intentions is an effective selfregulation strategy for promoting goal attainment (see Wieber, Thürmer, \& Gollwitzer, 2015). However, the question of the influence of implementation intentions on effort-related cardiac activity during task performance is still unanswered.

Using an if-then plan has for instance been shown to help attaining long-term academic goals (Duckworth, Grant, Loew, Oettingen, \& Gollwitzer, 2011), to reduce self-handicapping (Thürmer, Mc Crea, \& Gollwitzer, 2013), to help decrease the consumption of unhealthy snacks (Adriaanse, de Ridder, \& de Wit, 2009), increase vitamin intake (Sheeran \& Orbell, 1999), eat a healthy diet (Chapman \& Ermitage, 2012; Verplanken \& Faes, 1999), facilitate recycling behavior (Holland, Aarts, \& Langendam, 2006) as well as breaking old habits (e.g., unhealthy snacking) and creating new ones (Adriaanse, Gollwitzer, de Ridder, de Wit, \& Kroese, 2011). By linking an anticipated critical situation to a goal-directed response, implementation intentions have been found to increase both people's perceptual and behavioral readiness when the critical 
cue specified in the if-then plan is encountered. Forming implementation intentions leads to a heightened activation of the mental representation of the specified critical situational cue, thus increasing its cognitive accessibility (e.g., Achtziger, Bayer, \& Gollwitzer, 2012, Study 1; ParksStamm, Gollwitzer, \& Oettingen, 2007), and it also leads to "strategic automaticity." Once the critical situation is encountered, the specified goal-directed response is initiated immediately (Gollwitzer \& Brandstätter, 1997), efficiently (Brandstätter, Lengfelder, \& Gollwitzer, 2001), with low controllability (Achtziger et al., 2012, Study 2; Wieber \& Sassenberg, 2006), and low conscious involvement (Bayer, Achtziger, Gollwitzer, \& Moskowitz, 2009).

Although many studies by now have demonstrated the positive influence of implementation intentions on task performance and, in addition, delineated the underlying cognitive processes, there is no direct evidence for the effects of implementation intentions on effort-related cardiac activity during task performance. Therefore, the aim of the present research is to investigate the influence of implementation intentions on effort mobilization during task performance.

\section{Effort-related Cardiovascular Response}

According to the Motivational Intensity Theory (Brehm \& Self, 1989), effort is mobilized proportionally to subjectively experienced task demand as long as success is possible and justified. This model, based on the principle of conserving resources, posits that individuals avoid wasting energy and thus mobilize resources proportionally to subjective task demand as long as success appears possible and attaining it justifies the mobilization of effort. On the basis of this psychophysiological literature (Obrist, 1981; Kelsey, 2012; Wright, 1996), we quantify effort mobilization by performance-related changes in cardiac contractility force regarding the cardiac pre-ejection period (PEP). 
Based on Wright's (1996) integration of the Motivational Intensity Theory (Brehm \& Self, 1989) and Obrist's (1981) active coping approach, it has been demonstrated that betaadrenergic receptor activity is directly proportional to the level of experienced task demand, given that success is perceived as possible and effort seems justified. In a non-invasive way, the increase of beta-adrenergic receptor activity relates to the increase of cardiac contractility. A strong cardiac contractility indicates a higher effort mobilization. Among the different cardiac indicators, the increase of the cardiac contractility speaks to a shortened cardiac PEP. The cardiac pre-ejection period is defined as the time interval between the onset of the heart's left ventricular excitation and the opening of the aortic valve (Berntson, Lozano, Chen, \& Cacioppo, 2004). This cardiac index (measured in milliseconds) refers directly to the cardiac contractility (the inherent strength of the heart's contraction) of the left ventricle before blood is ejected into the vasculature system. Cardiac PEP is directly influenced by $ß$-adrenergic impact, and is the most reliable indicator of the cardiac contractility force, and thus of effort mobilization during task performance (Kelsey, 2012).

In support of Wright's integrative model (Wright, 1996), cardiac PEP sensitively responds to variations in task difficulty (Freydefont, Gendolla, \& Silvestrini, 2012; Richter, Friedrich, \& Gendolla, 2008; Richter, 2012; Richter, Baeriswyl, \& Roets, 2012; Silvestrini \& Gendolla, 2013). Various studies also assessed effort in terms of reactivity of systolic blood pressure (SBP), systematically influenced by cardiac contractility through its impact on cardiac output (see Gendolla \& Richter, 2010; Wright \& Kirby, 2001). However, both systolic and diastolic blood pressure (DBP) are also influenced by peripheral vascular resistance, which is not systematically influenced by $\beta$-adrenergic impact (Levick, 2003) and can thus mask contractility effects on SBP and DBP. Still other studies (e.g., Eubanks, Wright, \& Williams, 2002) have quantified effort in terms of heart rate response (HR). However, HR is influenced by both sympathetic and 
parasympathetic activity of the heart and thus only reflects effort mobilization if the sympathetic activity is stronger than the parasympathetic activity (Berntson et al., 2004). In sum, of all of the indicators of effort intensity listed above, cardiac PEP is considered to be the most reliable and valid (Kelsey, 2012). Nevertheless, cardiac PEP should always be assessed together with blood pressure and HR to control for possible pre-load (ventricular filling) or after-load (arterial pressure) effects (Sherwood et al., 1990).

\section{The Present Research}

Based on both previous research on forming goal and implementation intentions and the literature on effort mobilization during task performance, we conducted two experiments in order to investigate the moderating effect of goal and implementation intentions on effort-related cardiac activity during task performance. Goal intentions achieve their beneficial effects on goal attainment via the induction of a commitment to reach the goal at hand. As long as the goal is easy or only moderately difficult this commitment assures goal attainment (e.g., Locke \& Latham, 1990, 2013). However, when goal striving is very challenging, the forming of implementation intentions is needed to attain one's goals (Oettingen, 2012). Forming implementation intentions facilitates goal attainment by creating an automaticity regarding the initiation of goal-directed responses (summary by Gollwitzer, 2014). Once the critical situation specified in the implementation intention is encountered, goal-directed responses are initiated swiftly and efficiently (e.g., Brandstätter, Lengfelder, \& Gollwitzer, 2001). Therefore, based on Brehm and Self's theory (1989), we hypothesized that action control by implementation intentions (as it runs off in an effortless way) should reduce the perceived task demand and subsequent effort mobilization during task performance.

Experiment 1 investigates the influence of forming goal intentions and implementation intentions (compared to a control group with mere task instructions) on effort mobilization during 
the performance of an easy classification task (i.e., a straightforward number identification task). We predicted a lower effort-related cardiac activity in both the implementation intention and the goal intention conditions compared to the control condition. However, as perceived task demand is also influenced by the level of task difficulty (i.e., the more difficult the task, the higher the experienced task demand as long as success is possible and justified; Richter et al., 2008), we designed Experiment 2 to investigate the conjoint impact of task difficulty and having formed goal vs. implementation intentions on effort mobilization during a difficult and very difficult dual task. In line with the Motivational Intensity Theory (Brehm and Self, 1989), we predicted that participants in the goal intention condition should readily disengage when the task becomes very difficult, as success is no longer perceived as possible and thus effort mobilization does not seem to be justified anymore. In contrast, participants who formed implementation intentions should "stay in the field;" their effort-related cardiac activity should be higher in the very difficult task condition, as successful task performance still appears feasible and justified.

\section{Experiment 1: The Moderating Effect of Goal and Implementation Intentions on Effort-related Cardiac Activity during Easy Task Performance}

Past research on goals and plans shows that committing to easy goals facilitates goal attainment (Locke \& Latham, 1990, 2014). Moreover, making additional plans on how to reach these goals does not provide a further benefit (Gollwitzer \& Sheeran, 2006). Rather, forming mere goal intentions suffices to promote goal striving. Based on these findings and according to the Motivational Intensity Theory (Brehm \& Self, 1989), we posited that both the goal intention strategy and the implementation intention strategy should moderate task demand when having to perform an easy task, and as a consequence, should reduce effort mobilization during task performance. In order to provide evidence of a moderating effect of goal and implementation intentions on effort-related cardiac activity, an experiment composed of three conditions (goal 
intention, implementation intention, and control) was conducted. Assessing cardiovascular activity, SBP, DBP, HR, and especially cardiac PEP as a direct measure of effort mobilization during task performance, we predicted a weaker cardiac PEP reactivity in both the goal and the implementation intention conditions as compared to the control condition (where participants only received task instructions).

\section{Method}

Participants and design. Sixty healthy undergraduate psychology students (37 women, $\left.M_{\text {age }}=19.91, S D=1.38\right)$ from New York University were randomly assigned to one of 3 experimental conditions (goal intention vs. implementation intention vs. control). Participation in this experiment was voluntary and course credit was given for participation. However, data from 13 participants were excluded because of incomplete data due to technical problems (i.e., cardiac activity assessment was missing), resulting in a final sample of 47 participants.

Apparatus and physiological measures. We assessed cardiovascular activity with AcqKnowledge 4.2, Biopac System, Inc. software. Four disposable tapes were placed around the participant's neck and torso (at the height of the xiphoid), and 2 electrodes were placed on the torso. Electro-cardiac signals were assessed during the entire duration of the experiment with a sampling rate of $1000 \mathrm{~Hz}$ and analyzed with the BlueBox 2 v1.22 software (Richter, 2010). The first derivative of the change in thoracic impedance was calculated and the resulting dZ/dt-signal was averaged over periods of 1 min using the detected R-peaks (Kelsey \& Guethlein, 1990). To obtain cardiac PEP values (time interval in ms between R-onset and B-point, as defined by Berntson et al., 2004), B-point location was estimated based on the RZ interval as suggested by Lozano et al. (2007). Whenever necessary, the B-point location was manually corrected as recommended by Sherwood et al. (1990). Moreover, we assessed SBP and DBP with a Dinamap ProCare V100 monitor (GE Medical Systems Information Technologies Inc., Milwaukee, WI) 
which uses oscillometry. A blood pressure cuff (DuraCuf) was placed above the brachial artery of the participant's left arm. The cuff automatically inflated in one-minute intervals during habituation and task performance periods. Systolic and diastolic pressure values were recorded each minute during these two periods.

Material and procedure. The study was approved by the local ethics committee. In the present experiment, we used a classification task inspired by Brandstätter et al. (2001), in which participants had to distinguish single-digit numbers from multiple-digit numbers.

The digit classification task. Each of the 60 trials started with a fixation cross in the center of the screen for $1000 \mathrm{~ms}$ followed by a single or multiple digit number for $3000 \mathrm{~ms}$ maximum. Participants had to indicate whether this number was a single $(1,3,5,7$ or 9) or multiple digit number $(16,39,44,694$, or 555) by pressing the green button (single digit number) or the red button (multiple digit number) on the keyboard. Afterwards, the message "response entered" appeared. In case of no response, participants were shown the message: "Please answer more quickly!” To keep the task duration constant and avoid a habituation effect, this message appeared for $4 \mathrm{~s}$ minus the participant's reaction time. The inter-trial intervals randomly varied between $2000 \mathrm{~ms}$ and $5000 \mathrm{~ms}$. In each experimental condition, the number " 3 " was presented in $15 \%$ of the trials. $50 \%$ of the trials presented a single digit number, and $50 \%$ showed multiple digit numbers.

Procedure. The experiment was run in individual sessions. After having signed the informed consent form, participants were seated in a comfortable chair in front of a computer screen. First, the experimenter attached tapes, electrodes and the blood pressure cuff to the participant and then left the room. At the beginning of the experiment, participants answered simple biographical questions (age, smoker, family history of cardiovascular disease, medication influencing the cardiovascular system). Thereafter, we recorded the cardiovascular baseline 
activity ( $8 \mathrm{~min}$ ) during the habituation period. During this period, participants watched a documentary film of neutral valence about Lithuania (Freydefont \& Gendolla, 2012).

Finally, participants received instructions for the digit classification task, followed by a "Please respond as quickly and accurately as possible!" No information regarding the task difficulty was given in order to keep the level of difficulty unclear. After a training session composed by 10 trials, supplementary instructions according to the experimental condition appeared on the screen for a duration of two minutes. In the goal intention condition, participants were instructed to tell themselves: "I will press the green key especially quickly when the number 3 appears!" In the implementation intention condition, participants were instructed to tell themselves: "IF the number 3 appears, THEN I will press the green key especially quickly!" Participants in the control condition were instructed to look at the number 3: "Please, look at the number 3 !" Then participants performed the 60 trials of the digit classification task.

After performing the task, participants retrospectively rated the perceived subjective task difficulty, ability to succeed, amount of mobilized effort, and the importance of success on 7point scales ranging from 1 (not at all) to 7 (very much). We did this to check whether the various task instructions given to participants in the three conditions differentially affected the subjective perception of certain features of task performance. At the end of the experiment, participants were debriefed, thanked, and given course credit for their participation.

\section{Results}

Cardiovascular baselines. Baseline values for cardiac PEP, SBP, DBP, and HR were calculated by averaging cardiovascular values from the last three minutes of the habituation period (all Cronbach's $\alpha \mathrm{s}>$.92). Means and standard errors are depicted in Table 1. One-way ANOVAs revealed no significant effects of conditions on any cardiovascular index (all $p \mathrm{~s}>.58$ ). Also, statistical analyses indicated that there was no gender effect on any cardiovascular baseline 
values $(p s>.08)$ except for the systolic blood pressure. Compared to women, men had higher SBP (respectively $M=113.70, S E=1.28$ and $M=100.02, S E=1.56), F(1,46)=40.38, p<.01$, $\eta_{p}{ }^{2}=0.48$, which is consistent with previous findings (Wolf et al., 1997). Accordingly, we adjusted for gender in the statistical analyses regarding SBP reactivity.

Cardiovascular reactivity. Cardiovascular reactivity scores for cardiac PEP, SBP, DBP, and HR were determined by subtracting cardiovascular baseline from the averaged 1 min scores of cardiovascular activity during task performance (all Cronbach's $\alpha$ s $>.94$ ). This means that a high negative score indicates high effort mobilization, whereas a low negative score indicates low effort. Preliminary analyses observed no significant gender effects on any cardiovascular index $(p s>.18)$.

Cardiac PEP reactivity. According to our hypothesis, we conducted an a priori contrast analysis (goal intention: -1 , implementation intention: -1 , control condition: 2 ) showing a significant main effect of goal striving strategies on cardiac PEP reactivity, $t(44)=3.49, p<.002$, $d=1.05$. Also, as depicted in Figure 1, focused cell comparisons showed a significant weaker cardiac PEP reactivity in the goal intention condition $(M=1.47, S E=1.43)$ than in the control condition $(M=-7.03, S E=2.21), t(44)=3.51, p<.001, d=1.06$. Moreover, cardiac PEP reactivity in the implementation intention condition was also significantly weaker $(M=-0.84, S E$ $=1.37)$ than in the control condition $(M=-7.03, S E=2.21), t(44)=2.51, p<.001, d=0.75$. No significant difference was found between the goal intention and the implementation intention condition $(p>.35)$.

SBP, DBP, and HR reactivity. When the same a priori contrast analysis was conducted for SBP, DBP, and HR, it did not reveal any significant effects ( $p$ s > .45). Moreover, we conducted an ANCOVA on the SBP reactivity with gender as covariate and found no significant gender effect $(p>.24)$. Means and standard errors are depicted in Table 2. 
Task performance. As recommended (Winer, 1971), extreme reaction time (RT) outliers (i.e., RTs more than 2 standard deviations above and below the mean of each response) were excluded. Moreover, RTs were natural log transformed before entering them into the analyses, as these variables were positively skewed. However, for ease of interpretation, all reported mean scores and standard deviations are presented with the non-transformed RTs (for a similar approach see Custers \& Aarts, 2007; De Houwer, 2003; Kroese, Evers, \& De Ridder, 2011). As participants were asked to focus on the number " 3 ," our critical performance analysis pertained to the target stimulus " 3 ." Participants in the goal intention condition $(M=405.53, S E=10.45)$ and the implementation intention condition $(M=400.64, S E=13.76)$ were faster than participants in the control condition $(M=475.16, S E=18.09)$, overall $F(2,44)=6.80, p<.004, \eta_{p}{ }^{2}=0.27$. More specifically, planned comparisons between conditions revealed that participants in the goal intention condition were significantly faster than participants in the control condition, $t(44)=$ 3.44, $p<.001, d=0.93$. A similar pattern was observed for participants in the implementation intention condition, $t(44)=3.62, p<.001, d=0.98$; participants in the implementation intention condition were significantly faster than participants in the control condition. No significant difference was found between the goal intention condition and implementation intention condition $(p=.96)$. Also, we observed no significant effect of condition on the percentage of correct responses regarding the target number“3," (overall $M=92.13, S E=0.21$ ), $p=.38$.

A three-condition one-way ANOVA revealed a significant main effect of condition on response times (in ms) for all of the numbers (excluding the number " 3 ”), $F(2,44)=3.62, p=$ $.03, \eta_{p}{ }^{2}=0.14$. Participants in both the goal and implementation intention condition responded significantly faster $(M=425.67, S E=11.73$ and $M=427.49, S E=14.20$, respectively $)$ than participants in the control condition $(M=476.69, S E=18.55)$. Planned comparisons showed a significant difference between the goal intention and the control condition, $t(44)=2.35, p<.001$, 
$d=0.71$, as well as between the implementation intention and the control condition, $t(44)=2.29$, $p<.001, d=0.69$. We observed no significant effect of the goal striving strategies between the 3 experimental conditions regarding the percentage of correct responses (excluding the responses to the number "3"), $(M=94.17, S E=0.01), p>.37$.

Task ratings. ANOVAs on participants' ratings regarding task difficulty $(M=1.95, S E=$ $0.19)$, mobilized effort $(M=2.65, S E=0.23)$, the subjective value of success $(M=3.77, S E=$ $0.21)$, and the ability to succeed $(M=3.70, S E=0.27)$ revealed no significant effects of condition (all $p s>$.27). This suggests that the differences observed between conditions in terms of effort mobilization cannot be attributed to differential subjective perception of these features of task performance.

\section{Discussion}

In Experiment 1, we investigated the influence of goal intentions and implementation intentions on effort-related cardiac response during easy task performance. A total of 47 university students were randomly assigned to one of three conditions (goal intention vs. implementation intention vs. control) in a between-participants design. Based on numerous studies showing the effectiveness of both forming goal and implementation intentions on attaining easy goals and stimulated by the Motivational Intensity Theory (Brehm \& Self, 1989), we suggested that both goal intentions and implementation intentions should reduce task demand and thus effort mobilization during easy task performance. As predicted, we observed a weaker cardiac PEP in both goal and implementation intention conditions than in the control condition. Moreover, results regarding performance in term of response times revealed a better performance in both the goal intention and the implementation intention conditions as compared to the control condition. 
Both participants in the goal intention and in the implementation intention conditions performed better than participants in the control condition. This finding is due to the fact that the chosen digit classification task (i.e., one digit numbers vs. multiple digit numbers) was very easy. Indeed, research on implementation intentions consistently shows that the beneficial effects of forming implementation intentions on goal attainment in comparison to mere goal intentions accrue only when the task goal at hand is difficult to reach (Gollwitzer, 2014). Moreover, according to the principle of energy conservation posited by Brehm and Self in the Motivational Intensity Theory (1989), people mobilize only the level of effort needed during task performance, not more. Accordingly, the low level of cardiac PEP reactivity in both goal intention and implementation intention conditions regarding effort mobilization suggests that low task difficulty resulted in a ceiling effect regarding performance; both goal intention and implementation participants managed to do very well on the task with little effort mobilization. Accordingly, in Experiment 2 we decided to establish two different levels of task difficulty: difficult versus very difficult.

Experiment 2: Task Difficulty and Goal Striving Strategies: Influence of Goal and

\section{Implementation Intentions on Effort-related Cardiac Activity during Task Performance}

Experiment 2 manipulated 2 levels of task difficulty, difficult and very difficult, and assessed effort-related cardiac activity during task performance. We hypothesized that in the difficult task condition, participants with implementation intentions will mobilize a lower amount of effort than both participants with mere goal intentions and control participants. Because participants in the implementation intention condition should experience the task as rather easy (because of the efficiency associated with action control by implementation intentions), they should exert only the low amount of effort necessary for them to succeed at a difficult task. In contrast, participants in the goal intention condition should evaluate the difficult task as 
challenging and therefore mobilize a large amount of effort matching a high level of difficulty. However, in the very difficult task condition, participants with implementation intentions should mobilize more effort than participants with goal intentions and participants in the control condition. Because the latter participants should experience the task as too difficult, they should see high effort mobilization as no longer justified, and thus these participants (i.e., goal intention and control condition participants) should disengage. In contrast, participants with implementation intentions should experience the very difficult task as still possible, and thus they should increase their effort to match effort mobilization to the high degree of perceived demand in order to succeed.

\section{Method}

Participants and design. Ninety healthy undergraduate psychology students from New York University ( 55 women, $M_{\text {age }}=19.58, S D=1.21$ ) were randomly assigned to a 3 goal striving strategies (goal intention vs. implementation intention vs. control) x 2 levels of task difficulty (difficult vs. very difficult) between-participants design. Data from 12 participants were excluded from statistical analyses because of incomplete data, and 10 participants were excluded for technical problems making the obtained physiological data non-usable; this resulted in a final sample of 72 participants ( 41 women, $M_{\text {age }}=19.53, S D=1.23$ ). Participation was voluntary and course credit was given for participation.

Apparatus and physiological measures. We assessed cardiovascular activity with the identical material as in Experiment 1, AcqKnowledge 4.2, Biopac System, Inc. software. Electrocardiac signals were assessed during the entire experiment with a sampling rate of $1000 \mathrm{~Hz}$ and analyzed with the BlueBox 2 v1.22 software (Richter, 2010) as in Experiment 1. Moreover, we assessed SBP and DBP with a Dinamap ProCare V100 monitor (GE Medical Systems 
Information Technologies Inc., Milwaukee, WI). Systolic and diastolic pressure reactivity scores were recorded each minute during the habituation period and the task.

Materials and procedure. The present procedure was approved by the local ethics committee. We used a dual-task paradigm adopted from Wieber, Sezer, and Gollwitzer (Study 2, 2014).

The dual-task paradigm. Because multi-tasking is commonly experienced as very demanding (e.g., Appelbaum et al., 2008; Czerwinski et al., 2004), we decided to use a dual task paradigm combining a tracking task with a go/no go classification task in Experiment 2. Participants had to cover a circle $(1.7 \mathrm{~cm}$ diameter $)$ moving on a random course across the computer screen with a larger mouse-controlled second circle. We manipulated the level of task difficulty by controlling the size of the mouse-circle $(4.2 \mathrm{~cm}$ diameter in the difficult task condition vs. $2.3 \mathrm{~cm}$ diameter in the very difficult task condition). In addition to this tracking task, participants had to perform a go/no-go classification task. In this additional task, participants had to discriminate letters from numbers by pressing the left mouse button whenever numbers appeared in the target circle and to refrain from pressing any button when a letter appeared. As the instructions for the tracking and the classification task were provided at the same time and did not mention a primary or a secondary task, we focused on the reaction times of correct pressing responses as the dependent variable.

Procedure. As in Experiment 1, the procedure started with the signing of the consent form and the preparation of the cardiovascular assessment, followed by the biographical questions and the recording of the cardiovascular baseline activity ( $8 \mathrm{~min}$ ) during the habituation period. Then participants received instructions for the dual task accompanied by the quote “Please respond as quickly and accurately as possible!" As in Experiment 1, no information regarding the level of task difficulty was given. After the training session, supplementary 
instructions specific to the experimental condition appeared on the screen for a duration of two minutes. In the goal intention condition, participants were instructed to tell themselves: "I will click the left mouse button very quickly when a number appears!” In the implementation intention condition, participants were instructed to tell themselves: "IF a number appears, THEN I will click the left mouse button very quickly!" Participants in the control condition were instructed to look for numbers: "I will pay particular attention to the numbers!" Then participants performed the 48 trials of the dual task ( 6 min). After finishing the task, participants retrospectively rated subjective task difficulty, ability to succeed, amount of mobilized effort, and the importance of success on 7-point scales ranging from 1 (not at all) to 7 (very much). Finally, participants were debriefed, thanked, and given course credit for their participation.

\section{Results}

Cardiovascular baselines. Baseline values were calculated by averaging cardiovascular values from the last $3 \mathrm{~min}$ of the habituation period (all Cronbach's $\alpha \mathrm{s}>.94$ ). Cell means and standard errors are depicted in Table 3. A 2 X 3 ANOVA found neither significant effects of the goal striving strategies factor nor an effect of the task difficulty factor on any cardiovascular index $(p s>.11)$. Preliminary statistical analyses indicated that there was no gender effect on any cardiovascular baseline values $(p s>.16)$ except for the SBP and HR. As in Experiment 1, compared to women, men had higher $\operatorname{SBP}(M=114.41, S E=1.58$ vs. $M=103.71, S E=1.20)$, $F(1,70)=30.24, p<.001, \eta_{\mathrm{p}}{ }^{2}=.30$. Moreover, we also observed that men had higher HR $(M=$ 80.72, $S E=1.95)$ than women $(M=74.42, S E=2.12), F(1,70)=4.74, p<.04, \eta_{\mathrm{p}}{ }^{2}=.06$, which is consistent with the respective research literature (Wolf et al., 1997); accordingly, we adjusted for gender in the further analyses on SBP and HR.

Cardiovascular reactivity. Cardiovascular reactivity scores for cardiac PEP, SBP, DBP, and HR were determined by subtracting cardiovascular baseline values from the averaged 1 min 
scores of cardiovascular activity during task performance (all Cronbach's $\alpha$ s $>$.94). Preliminary analyses found no significant gender effects on any cardiovascular indicators $(p s>.16)$. After having conducted conventional ANOVAs for each cardiovascular index, we tested our predictions by a priori contrast analyses.

Cardiac PEP reactivity. First, we conducted an a priori contrast analysis in accordance with our predictions. In order to demonstrate the moderating effect of implementation intentions on effort-related cardiac activity in Experiment 2, we used a dual task paradigm that clearly qualifies as a difficult task in comparison to the easy task used in Experiment 1. Given that the dual task in Experiment 2 has to be considered to be more difficult than the digit number task of Experiment 1, we had to adjust our predictions concerning the implementation intention and goal intention conditions in the difficult task condition. Accordingly, we specified the contrast weights as follows: goal intention/difficult: -1 , implementation intention/difficult: 2 , control/difficult: - 1 , goal intention/very difficult: 1 , implementation intention/very difficult: -2 , control/very difficult: 1). Results showed a significant joint effect of goal striving strategies and task difficulty on the cardiac PEP, $t(66)=2.24, p<.03, d=0.55$. As depicted in Figure 2, in the difficult task condition we observed a weaker cardiac PEP reactivity for the implementation intention participants $(M=$ 0.37, $S E=2.19)$ as compared to both goal intention $(M=-2.78, S E=1.82)$ and control participants $(M=-2.42, S E=1.29)$. Importantly, for the very difficult task condition, we observed that participants with implementation intentions increased effort $(M=-2.18, S E=1.56)$ compared to the difficult task condition $(M=0.37, S E=2.19)$. In contrast, participants in the goal intention and control conditions showed the opposite pattern (respectively, $M=4.28, S E=2.01$ and $M=-1.25, S E=1.06$ ). Supporting our hypothesis regarding the moderating effect of implementation intentions on effort-related cardiac activity, planned comparisons indicated that in the very difficult task condition, the cardiac PEP response in the implementation intention 
condition was significantly stronger than in the goal intention condition, $t(66)=2.77, p<.01, d=$ 0.68. We also observed a significant difference between the goal intention/difficult task condition $(M=-2.78, S E=1.82)$ and goal intention/very difficult task condition $(M=4.28, S E=2.01)$, revealing a weaker cardiac PEP reactivity in the goal intention/very difficult task condition, $t(66)$ $=2.74, p<.01, d=0.67$. Finally, no significant difference was found between the implementation intention condition and the goal intention condition in the difficult task condition $(p>.11)$. There were also no significant main effects of the goal striving strategy or the task difficulty manipulations $(p s>.21)$.

SBP, DBP and HR reactivity. For SBP, DBP, and HR reactivity, we conducted the same statistical analyses as for cardiac PEP reactivity. The respective a priori contrasts showed no significant interaction or main effects on all of these measures ( $p s>.62)$ (see Table 4).

Task performance. As recommended (Winer, 1971), extreme reaction time (RT) outliers (i.e., more than 2 standard deviations above and below the mean of reaction times) were excluded. Moreover, RTs were natural log transformed before entering them into the analyses, as this variable was positively skewed. However, for ease of interpretation, all reported mean scores and standard deviations are depicted with the non-transformed variables as in Experiment 1 . We conducted a 2 X 3 ANOVA on the response times for the correct responses (when participants pressed the left mouse button for numbers). Results revealed no significant main or interaction effects of goal striving strategy and task difficulty, $p s>.63$. Moreover, we conducted the same analysis for the percentage of correct responses (i.e., pressing for numbers but not letters). Results revealed no significant main or interaction effects for goal striving strategy and task difficulty on the percentage of correct responses $(M=88.30 \%, S E=2.25)$, ps $>.19$.

Task ratings. A 3 X 2 ANOVA revealed a significant interaction between goal striving strategy and task difficulty on the subjective evaluation of task difficulty, $F(2,71)=8.01, p<$ 
$.01, \eta_{\mathrm{p}}{ }^{2}=.19$ (see Figure 3 ). Findings showed that in the difficult task condition, participants in the goal intention condition $(M=0.90, S E=0.46)$ evaluated the task as easier than participants in the control condition $(M=1.93, S E=0.37), t(66)=1.74, p<.05, d=0.43$. We neither observed significant differences between the implementation intention and goal intention conditions, nor between the implementation intention and control conditions $(p s>.15)$. We also found that in the difficult task condition, participants in the implementation intention condition evaluated the task as more difficult $(M=2.33, S E=0.35)$ than participants in the control condition $(M=0.25, S E=$ $0.16), t(66)=3.26, p<.01, d=0.80$. We observed the same pattern when comparing the goal intention condition $(M=2.58, S E=0.42)$ with the control condition $(M=0.25, S E=0.16), t(66)$ $=3.51, p<.01, d=0.86$. There was no significant difference in the ratings of task difficulty between the implementation intention and goal intention condition participants $(p>.32)$. Finally, planned comparisons regarding rated task difficulty between conditions revealed that participants in the implementation intention and goal intention conditions evaluated the difficult task as easier (respectively, $M=1.33, S E=0.41$ and $M=0.90, S E=0.45$ ) than the difficult task (respectively, $M=2.33, S E=0.35$ and $M=2.58, S E=0.42), t(66)=1.77, p<.01, d=0.43$ and $t(66)=3.51, p$ $<.01, d=0.66$. However, we observed that participants in the control condition rated the difficult task $(M=1.93, S E=0.48)$ as more difficult than the very difficult task $(M=0.25, S E=0.16)$, $t(66)=2.64, p<.01, d=0.65$. Moreover, no significant main effects of goal striving strategy and task difficulty or a significant interaction effect was found for the self-evaluation of ability $(M=$ 3.99, $S E=0.23)$, reported effort mobilization $(M=3.28, S E=0.20)$, and importance of success $(M=3.58, S E=0.19)$, all $p s>.10$. In sum, the patterns observed for the variables describing subjectively perceived task features do not match the patterns observed for cardiac PEP and thus do not qualify as potential alternative explanations.

\section{Discussion}


Experiment 2 investigated the joint impact of goal striving strategies and the level of task difficulty on effort-related cardiac activity during task performance. Results showed a significant interaction between the two levels of task difficulty (difficult vs. very difficult) and goal striving strategy (goal intention vs. implementation intention vs. control). In the difficult task condition, we observed a weaker cardiac PEP reactivity in the implementation intention condition than in the goal intention and control conditions, indicating a less pronounced effort mobilization in the implementation intention group as compared to the other two experimental groups. Importantly, we observed the opposite pattern in the very difficult task condition. We found a stronger cardiac PEP reactivity in the implementation intention condition than in the goal intention and control conditions, demonstrating a more pronounced effort mobilization in the implementation intention condition than in the goal intention and control conditions. This pattern of findings suggests that although participants in the implementation intention condition evaluated the task as difficult, they experienced it as feasible and increased effort accordingly to succeed in the more challenging task. In contrast, participants in the goal intention condition experienced the task as too demanding; they saw further effort mobilization as unjustified and as a consequence disengaged (Brehm \& Self, 1989).

\section{General Discussion}

The present line of research investigated the influence of forming goal and implementation intentions on effort mobilization during task performance. Based on studies demonstrating the beneficial effects and processes of goal and implementation intentions regarding goal attainment on the one hand, and the hypotheses posited by the Motivational Intensity Theory (Brehm \& Self, 1989) on the other hand, we suggested that the self-regulation strategies of forming goal and implementation intentions should moderate effort mobilization during task performance. Together, Experiments 1 and 2 confirmed our predictions, showing a 
moderating effect of making an if-then plan on effort mobilization during task performance. Experiment 1 investigated the impact of forming goal and implementation intentions on effortrelated cardiac activity compared to acting on mere task instructions (control group) when an easy cognitive task had to be performed (i.e., a simple number classification task). Results from Experiment 1 revealed a significant shorter cardiac PEP in the control condition, indicating a more pronounced effort mobilization than in both the goal intention and the implementation intention condition (Figure 1). Experiment 2 investigated the joint impact of goal striving strategies and task difficulty on effort mobilization using a challenging dual task paradigm. We observed a significant interaction between goal striving strategy and task difficulty on cardiac PEP reactivity. Whereas participants in the goal intention condition disengaged under the very difficult task condition, participants in the implementation intention condition increased effort mobilization in line with task difficulty.

In the present research, cardiac PEP reactivity (our primary dependent variable assessing effort mobilization during task performance; Kelsey, 2012; Wright, 1996) was always assessed together with blood pressure and HR to control for possible pre-load (ventricular filling) or afterload (arterial pressure) effects (Sherwood et al., 1990). In Experiments 1 and 2, SBP, DBP and HR reactivity showed a similar but not significant pattern as observed for cardiac PEP reactivity. The absence of significant effects on SBP, DBP and HR reactivity is not problematic, however, as SBP and DBP are also influenced by alpha-adrenergic peripheral resistance in the vasculature, which can mask the influence of beta-adrenergic impact on blood pressure (see Levick, 2003). Moreover, pre-load and after-load effects are mostly observed when participants are in motion. In studies investigating effort intensity in cognitive tasks, during which the body is in a stable position, pre-load effects are very unlikely (Bernston, Cacioppo, \& Quigley, 1993; Cacioppo, Berntson, Larsen, Poehlmann, \& Ito, 2000; Kelsey, 1991). As a consequence, SBP, DBP and HR 
do not always correspond to effort mobilization (Freydefont \& Gendolla, 2012; Freydefont et al., 2012; Silvestrini \& Gendolla, 2013). As pointed out by Silvestrini and Gendolla (2013, Experiment 1), if neither HR nor blood pressure decreases significantly when cardiac PEP becomes shorter, effects found on cardiac PEP reactivity can be attributed to beta-adrenergic impact rather than pre-load (ventricular filling) or after-load (vascular resistance) effects. Accordingly, our findings from Experiments 1 and 2 revealed, as expected, a significant impact of goal striving strategies on effort-related cardiac activity during task performance.

Furthermore, our results from Experiment 1 showed a significant effect of goal striving strategies on the performance measure of response time. Participants in the goal and the implementation intention conditions were significantly faster than participants in the control condition. Forming goal intentions can be assumed to create a strong commitment to attain the goal at hand (Locke \& Latham, 1990, 2013), whereas implementation intentions can be assumed to lead to a strong associative link between the critical situational cue and the specified goaldirected response (Gollwitzer, 1999, 2014; Gollwitzer \& Oettingen, 2011). As the task in Experiment 1 was very easy, we did not observe a significant difference between the goal and implementation intention conditions in reaction times. This is in line with past research on goal striving showing that performing well on easy tasks does not need action control by if-then plans; guidance by mere goal intentions suffices to promote goal attainment (Gollwitzer \& Sheeran, 2006; Gollwitzer, 2014).

As we predicted, in Experiment 1 results regarding the cardiac PEP in the goal intention condition indicated a significant effect on effort mobilization during task performance. As, goal intentions do not create a strong link between a specific situation and a goal-directed behavior, we cannot attribute the effect of the goal intention strategy to an automated behavioral response as assumed for the implementation intentions strategy ("I intend to perform behavior X!"). Only 
with the respect to the influence of implementation intentions on effort mobilization during task performance, we maintain that such effect are due to the automated responding triggered by linking the anticipated critical situation to the goal-directed behavior.

Regarding the effects of the goal intention strategy on effort mobilization during a cognitive task, it is unclear if it is due to a moderating effect of the subjective evaluation of task difficulty or a moderating effect due to the potential motivation. Indeed, an alternative explanation could be seen in the influence of the goal intention strategy on the potential motivation. The goal intention strategy, by increasing the potential motivation (Brehm \& Self, 1989), should lead to an increase of effort mobilization during task performance as long as success is possible and justified. As demonstrated in past research, potential motivation (defined as the maximum amount of effort justified by task success) is influenced by the importance of success when the task difficulty is unknown (see Richter, Gendolla, \& Wright, in press). This consideration is consistent with the absence of significant differences between the implementation intention and the goal intention condition regarding the ratings of task difficulty.

As observed in Experiment 1, in Experiment 2 we also did not observe any significant difference in reaction times between implementation intention and goal intention participants, even though this time participants worked on difficult and very difficult tasks and past research shows that implementation intentions do benefit the performance on difficult tasks. Although non-significant results have to be interpreted with caution, one possible interpretation of this null finding is that the performance on the dual task paradigm used heavily depends on people's willingness and ability to double task as well as their experience with double tasking.

Ratings assessed at the end of Experiment 1 on experienced effort mobilization, ability, importance of success, value of success, and task difficulty revealed no significant influence of goal striving strategy on the post-task self-report evaluations. One possible explanation of these 
results is that the measures taken after performance suffered from a number of biases that are typical for retrospective measures (see Robinson \& Clore, 2002). Importantly, in Experiment 2, we observed a significant interaction between goal striving strategy and task difficulty on the subjective ratings of task difficulty. According to our results, participants in the control group evaluated the task as less difficult than participants in the implementation intention and goal intention conditions (Figure 3). This could explain why participants in the control group did not disengage but showed a higher effort mobilization than participants in the goal intention condition. Possibly, control participants managed to "stay in the field" by convincing themselves that the task at hand is not that difficult after all. However, only participants with implementation intentions increased their amount of effort in the very difficult task as compared to the difficult task. Apparently, participants receiving goal intention instructions disengaged when performing the very difficult task, while participants with implementation intention instructions automatically increased their effort expenditure.

With regard to effort mobilization in an easy task context (Experiment 1) assessed by cardiac PEP, the goal intention strategy of "I intend to reach Z! had a similar impact on effort investment as the implementation intention strategy (see Figure 1). However, the significant interaction observed between goal striving strategy and task difficulty in Experiment 2 (see Figure 2) suggests that implementation intentions evince higher efficiency than goal intentions in the face of high task difficulty. This is in line with past implementation intention research consistently indicating that differences in action control by goal vs. implementation intentions emerge in particular when the task is difficult (Gollwitzer, 2014; Weeb \& Sheeran, 2006). Apparently, the automatic action control produced by implementation intentions leads to effortless performance. 
To conclude, our findings provide evidence that forming both goal and implementation intentions moderates effort mobilization during task performance. According to previous studies, goal intentions positively affect goal commitment and thus enhance goal striving (Locke \& Latham, 1990, 2013), but only as long as goal striving does not encounter challenging difficulties (e.g., obstacles encountered in multi-tasking; Oettingen, 2012). It is then when implementation intentions are needed, as this type of intentions automates action control and thus allows for efficient task performance (Achtziger et al., 2012; Brandstätter et al., 2001; Wieber et al., 2013). Based on our present results, it appears that the influence of implementation intentions does extend to effort mobilization during task performance as well. Whereas mere goal intentions may lead people to disengage when asked to perform very difficult tasks, implementation intentions allow people to "stay in the field" showing high effort expenditure.

\section{Acknowledgement}

We acknowledge the contributions of Sarang Park, Natasha Puri and Nathan Rennike to data collection.

This research was supported by a research grant from the Swiss National Science Foundation (P300P1-151192) awarded to Laure Freydefont and the Motivation Lab, New York University. 


\section{References}

Achtziger, A., Bayer, U. C., \& Gollwitzer, P. M. (2012). Committing to implementation intentions: Attention and memory effects for selected situational cues. Motivation and Emotion, 36, 287-303. doi: 10.1007//s11031-011-9261-6

Adriaanse, M. A., de Ridder, D. T. D., \& de Wit, J. B. F. (2009). Finding the critical cue: Implementation intentions to change one's diet work best when tailored to personally relevant reasons for unhealthy eating. Personality and Social Psychology Bulletin, 35, 6071. doi: $10.1177 / 0146167208325612$

Adriaanse, M. A., Gollwitzer, P. M., de Ridder, D. T. D., de Wit, J. B. F., \& Kroese, F. M. (2011). Breaking habits with implementation intentions: A test of underlying processes. Personality and Social Psychology Bulletin, 37, 502-513. doi:

$10.1177 / 0146167211399102$

Appelbaum, S. H., Marchionni, A., \& Fernandez, A. (2008). The multi-tasking paradox:

Perceptions, problems and strategies. Management Decision, 46, 1313-1325. doi:http://dx.doi.org/10.1108/00251740810911966

Bargh, J. A. (1992). The ecology of automaticity: Toward establishing the conditions needed to produce automatic processing effects. American Journal of Psychology, 105, 181-199. doi: $10.2307 / 1423027$

Bayer, U. C., Achtziger, A., Gollwitzer, P. M. \& Moskowitz, G. (2009). Responding to subliminal cues: Do if-then plans facilitate action preparation and initiation without conscious intent? Social Cognition, 27, 183-201. doi: 10.1521/soco.2009.27.2.183

Berntson, G. G., Cacioppo, J. T., \& Quigley, K. S. (1993). Cardiac psychophysiology and autonomic space in humans: Empirical perspectives and conceptual implications. Psychological Bulletin, 114, 296-322. doi: 10.1037/0033-2909.114.2.296 
Berntson, G. G., Lozano, D. L., Chen, Y. J., \& Cacioppo, J. T. (2004). Where to Q in cardiac PEP. Psychophysiology, 41, 333-337. doi: 10.1111/j.1469-8986.2004.00156.x

Brandstätter, V., Lengfelder, A., \& Gollwitzer, P. M. (2001). Implementation intentions and efficient action initiation. Journal of Personality and Social Psychology, 81, 946-960. doi: $10.1037 / / 0022-35 I 4.81 .5 .946$

Brehm, J. W., \& Self, E. A. (1989). The intensity of motivation. Annual Review of Psychology, 40, 109-131. doi: 10.1146/annurev.ps.40.020189.000545

Cacioppo, J. T., Berntson, G. G., Larsen, J. T., Poehlmann, K. M., \& Ito, T. A. (2000). The psychophysiology of emotion. In M. Lewis \& J. M. Haviland-Jones (Eds.), Handbook of emotions (2nd ed., pp. 173-191). New York: Guilford.

Czerwinski, M., Horvitz, E., \& Wilhite, S. (2004). A diary study of task switching and interruptions (pp. 175-182). ACM Press. doi:10.1145/985692.985715

Chapman, J., \& Armitage, C. J. (2012). Do techniques that increase fruit intake also increase vegetable intake? Evidence from a comparison of two implementation intention interventions. Appetite, 58, 28-33. doi: 10.1016/j.appet.2011.09.022

Custer, R., Aarts, H. (2007). In search of the nonconscious sources of goal pursuit: Accessibility and positive affective valence of the goal state. Journal of Experimental Social Psychology, 43, 312-318. doi: 10.1016/j.jesp.2006.02.005

De Houwer, J. (2003). A structural analysis of indirect measures of attitudes. In J. Musch \& K.C. Klauer (Eds.), The psychology of evaluation: Affective processes in cognition and emotion (pp. 219-244). Mahwah, NJ: Lawrence Erlbaum. 
Duckworth, A. L., Grant, H., Loew, B., Oettingen, G., \& Gollwitzer, P. M. (2011). Self-

regulation strategies improve self-discipline in adolescents: benefits of mental contrasting and implementation intentions. Educational Psychology, 31, 17-26. doi:

$10.1080 / 01443410.2010 .506003$

Eubanks, L., Wright, R. A., \& Williams, B. J. (2002). Reward influence on the heart:

Cardiovascular response as a function of incentive value at five levels of task demand.

Motivation and Emotion, 26, 139-152. doi: 10.1023/A:1019863318803

Faude-Koivisto, T. S., Wuerz, D., Gollwitzer, P. M. (2009). Implementation intentions: The mental representations and cognitive procedures of If-Then planning. In K. D. Markman, W. P. Klein, \& J. A. Suhr (Eds.) Handbook of imagination and mental simulation (pp. 6986). New York, NY: Taylor \& Francis group, Psychology Press.

Freydefont, L., \& Gendolla, G. H. E. (2012). Incentive moderates the impact of implicit anger versus sadness cues on effort-related cardiac response. Biological Psychology, 91, 120127. doi: 10.1016/j.biopsycho.2012.04.002

Freydefont, L., Gendolla, G. H. E., \& Silvestrini, N. (2012). Beyond valence: The differential effect of masked anger and sadness stimuli on effort-related cardiac response. Psychophysiology, 49, 665-671. doi: 10.1111/j.1469-8986.2011.01340.x

Gendolla, G. H. E., \& Richter, M. (2010). Effort mobilization when the self is involved: Some lessons from the cardiovascular system. Review of General Psychology, 14, 212-226. doi: $10.1037 / \mathrm{a} 0019742$

Gollwitzer, P. M. (1993). Goal achievement: The role of intentions. European Review of Social Psychology, 4, 141-185. doi: 10.1080/14792779343000059

Gollwitzer, P. M. (1999). Implementation intentions: Strong effects of simple plans. American Psychologist, 54, 493-503. 
Gollwitzer, P. M. (2014). Weakness of the will: Is a quick fix possible? Motivation and Emotion, 38, 305-322. doi: 10.1007/s11031-014-9416-3

Gollwitzer, P. M., \& Bargh, J. A. (2005). Automaticity in goal pursuit. In A. Elliot \& C. Dweck (Eds.), Handbook of competence and motivation (pp. 624-646). New York, NY: Guilford.

Gollwitzer, P. M., \& Brandstätter, V. (1997). Implementation intentions and effective goal pursuit. Journal of Personality and Social Psychology, 73, 186-199. doi:10.1037/00223514.73.1.186

Gollwitzer, P. M., \& Oettingen, G. (2011). Planning promotes goal striving. In K. D. Vohs \& R. F. Baumeister (Eds.), Handbook of self-regulation: Research, theory, and applications (2nd ed., pp. 162-185). New York, NY \London: Guilford Press.

Gollwitzer, P. M., \& Sheeran, P. (2006). Implementation intentions and goal achievement: A meta-analysis of effects and processes. Advances in Experimental Social Psychology, 38, 69-119. doi:10.1016/S0065-2601(06)38002-1

Holland, R. W., Aarts, H., \& Langendam, D. (2006). Breaking and creating habits on the working floor: A field-experiment on the power of implementation intentions. Journal of Experimental Social Psychology, 42, 776-783. doi: 10.1016/j.jesp.2005.11.006

Kelsey, R. M. (1991). Electrodermal lability and myocardial reactivity to stress. Psychophysiology, 28, 619-631. doi: 10.1111/j.1469-8986.1991.tb01005.x

Kelsey, R. M. (2012). B-adrenergic cardiovascular reactivity and adaptation to stress: The cardiac pre-ejection period as an index of effort. In R. A. Wright \& G. H. E. Gendolla (Eds.), How motivation affects cardiovascular response: Mechanisms and applications (pp. 4360). Washington DC: APA Press.

Kelsey, R. M., \& Guethlein, W. (1990). An evaluation of the ensemble averaged impedance cardiogram. Psychophysiology, 27, 24-33. doi: 10.1111/j.1469-8986.1990.tb02173.x 
Kroese, F. M., Evers, C., \& De Ridder, D. T. D. (2011). Tricky treats: Paradoxical effects of temptation strength on self-regulation processes. European Journal of Social Psychology, 41, 281-288. doi: 10.1002/ejsp.771

Levick, J. R. (2003). Introduction to cardiovascular physiology (4th ed). New York, NY: Oxford University Press.

Locke, E. A., \& Latham, G. P. (1990). A theory of goal setting and task performance. Upper Saddle River, NJ: Prentice Hall.

Locke. E. A., \& Latham, G. P. (2013) (Eds.), New developments in goal setting and task performance. New York, NY: Routledge.

Lozano, D.L., Norman, G., Knox, D., Wood, B. L., Miller, D., Emery, C. F., Bernston, G. C. (2007). Where to B in DZ/dt. Psychophysiology, 44, 113-119. doi: 10.1111/j.14698986.2006.00468.x

Obrist, P. A. (1981). Cardiovascular psychophysiology: A perspective. New York, NY: Plenum Press.

Oettingen, G. (2012). Future thought and behavior change. In W. Stroebe \& M. Hewstone (Eds.), European Review of Social Psychology, 23, 1-63. doi: 10.1080/10463283.2011.643698

Oettingen, G., \& Gollwitzer, P. M. (2010). Strategies of setting and implementing goals: Mental contrasting and implementation intentions. In J. E. Maddux \& J. P. Tangney (Eds.), Social psychological foundations of clinical psychology (pp. 114-135). New York, NY: Guilford Press.

Parks-Stamm, E. J., Gollwitzer, P. M., Oettingen, G. (2007). Action control by implementation intentions: Effective cue detection and efficient response initiation. Social Cognition, 25, 248-266. doi: 10.1521/soco.2007.25.2.248

Richter, M. (2010). BlueBox 2 v1.22 [computer software]. University of Geneva. 
Richter, M. (2012). Cardiovascular response to reward. In R. A. Wright \& G. H. E. Gendolla (Eds.), How motivation affects cardiovascular response: Mechanisms and applications (pp. 79-91). Washington DC: APA Press.

Richter, M., Baeriswyl, E., \& Roets, A. (2012). Personality effects on cardiovascular reactivity: Need for closure moderates the impact of task difficulty on engagement-related myocardial ß-adrenergic activity. Psychophysiology, 49, 704-707. doi: 10.1111/j.14698986.2011.01350.x

Richter, M., Friedrich, A., \& Gendolla, G. H. E. (2008). Task difficulty effects on cardiac activity. Psychophysiology, 45, 869-875. doi: 10.1111/j.1469-8986.2008.00688.x

Robinson, M. D., \& Clore, G. L. (2002). Belief and feeling: Evidence for an accessibility model of emotional self-report. Psychological Bulletin, 128, 934-960. doi: 10.1037/00332909.128.6.934

Sheeran, P., \& Orbell, S. (1999). Implementation intentions and repeated behavior: Augmenting the predictive validity of the theory of planned behavior. European Journal of Social Psychology, 29, 349-360. doi: 10.1111/j.1559-1816.1999.tb02298.x

Sherwood, A., Allen, M. T., Fahrenberg, J., Kelsey, R. M., Lovallo, W. R., \& van Dooren, L. J. P. (1990). Methodological guidelines for impedance cardiography. Psychophysiology, 27, 1-23. doi: 10.1111/j.1469-8986.1990.tb02171.x

Silvestrini, N., \& Gendolla, G. H. E. (2013). Automatic effort mobilization and the principle of resource conservation: One can only prime the possible and justified. Journal of Personality and Social Psychology, 104, 803-816. doi: 10.1037/a0031995

Thürmer, J. L., McCrea, S. M., \& Gollwitzer, P. M. (2013). Regulating self-defensiveness: Ifthen plans prevent claiming and creating performance handicaps. Motivation and Emotion, 37, 712-725. doi: 10.1007/s11031-013-9352-7 
Verplanken, B., \& Faes, S. (1999). Good intentions, bad habits, and effects of forming implementation intentions on healthy dieting. European Journal of Social Psychology, 29, 591-604. doi: 10.1002/(SICI)1099-0992(199908/09)29:5/6<591::AIDEJSP948>3.0.CO;2-H

Webb, T. L., \& Sheeran, P. (2006). Does changing behavioral intentions engender behavior change? A meta-analysis of the experimental evidence. Psychological Bulletin, 132, 249268. doi: 10.1037/0033-2909.132.2.249

Webb, T. L., \& Sheeran, P. (2008). Mechanisms of implementation intention effects: The role of goal intentions, self-efficacy, and accessibility of plan components. British Journal of Social Psychology, 47, 373-395. doi:10.1348/014466607X267010

Webb, T. L., Sheeran, P., \& Pepper, J. (2012). Gaining control over responses to implicit attitude tests: Implementation intentions engender fast responses on attitude-incongruent trials. British Journal of Social Psychology, 51, 13-32. doi:10.1348/014466610X532192

Wieber, F., \& Sassenberg, K. (2006). I can't take my eyes of it. Attention attraction effects of implementation intentions. Social Cognition, 6, 723-752. doi:

10.1521/soco.2006.24.6.723

Wieber, F., Sezer, L. A., \& Gollwitzer, P. M. (2014). Asking "Why" helps action control by goals but not plans. Motivation and Emotion, 38, 65-78. doi: 10.1007/s11031-013-9364-3

Wieber, F., Thürmer, L. J., \& Gollwitzer, P. M. (2015). Promoting the translation of intentions into action by implementation intentions: behavioral effects and physiological correlates. Frontiers in Human Neurosciences, 9:395. doi: 10.3389/fnhum.2015.00395

Winer, B. J. (1971). Statistical principles in experimental design ( $2^{\text {nd }}$ ed.). New York, NY: McGraw-Hill. 
Wolf, H. K., Tuomiletho, J., Kuulasmaa, K., Domarkiene, S., Cepaitis, Z., Molarius, A., Rywik, S. (1997). Blood pressure levels in the 41 populations of the WHO MONICA project. Journal of Human Hypertension, 11, 733-742. doi: 10.1038/sj.jhh.1000531

Wright, R. A. (1996). Brehm's theory of motivation as a model of effort and cardiovascular response. In P. M. Gollwitzer \& J. A. Bargh (Eds.), The psychology of action: Linking cognition and motivation to behavior (pp. 424-453). New York, NY: Guilford.

Wright, R. A., \& Kirby, L. D. (2001). Effort determination of cardiovascular response: An integrative analysis with applications in social psychology. Advances in Experimental Social Psychology, 33, 255-307. doi: 10.1016/S0065-2601(01)80007-1 


\section{Figure Captions}

Figure 1. Cell means and standard errors of cardiac pre-ejection period reactivity (in ms) during task performance in Experiment 1. Lower scores are indicating more effort expenditure during task performance.

Figure 2. Cell means and standard errors of cardiac pre-ejection period reactivity (in ms) during task performance in Experiment 2. Lower scores are indicating more effort expenditure during task performance.

Figure 3. Cell means and standard errors of the evaluation of the subjective task difficulty in Experiment 2. Higher scores are indicating higher perceived task difficulty. 
Figure 1

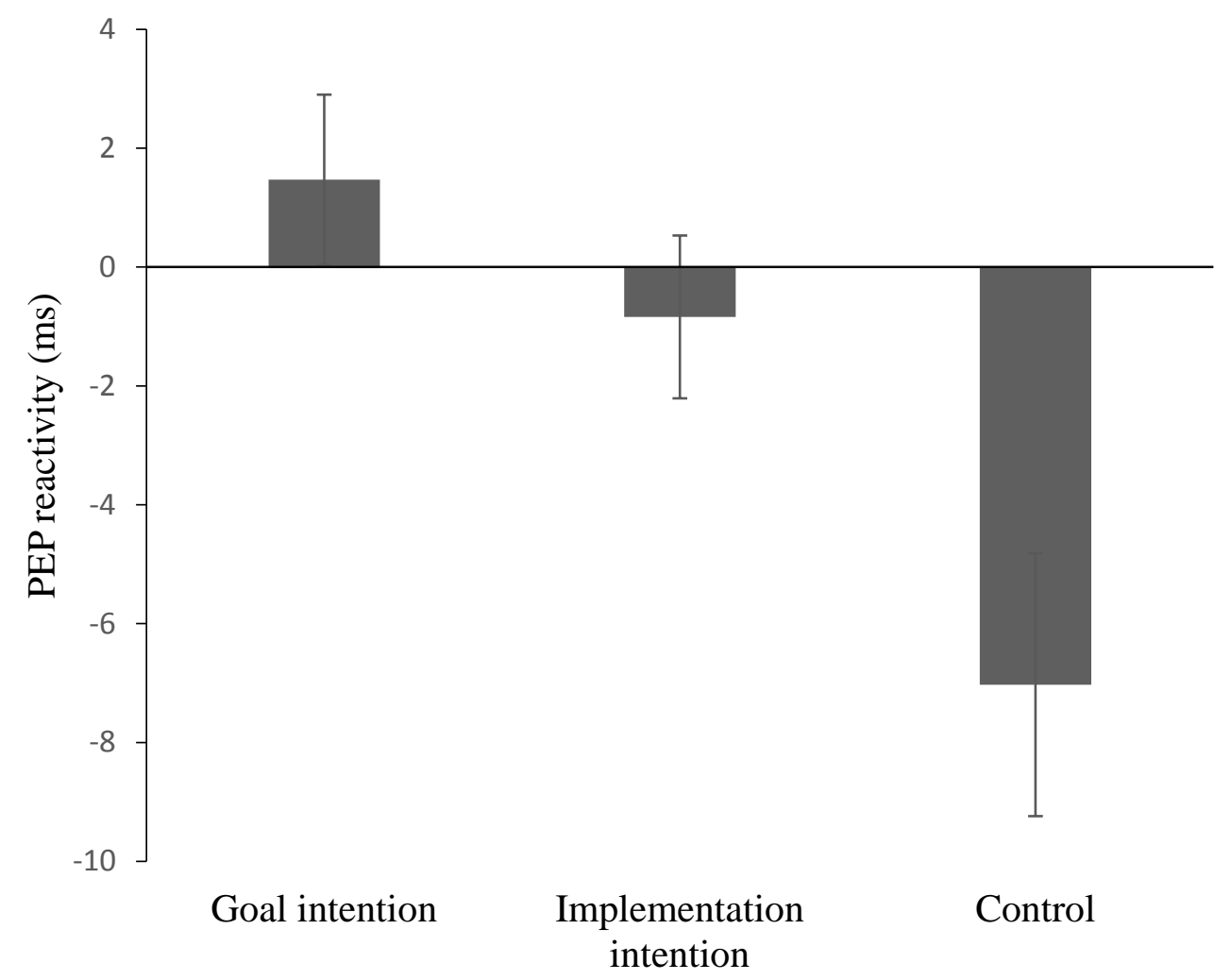


Figure 2

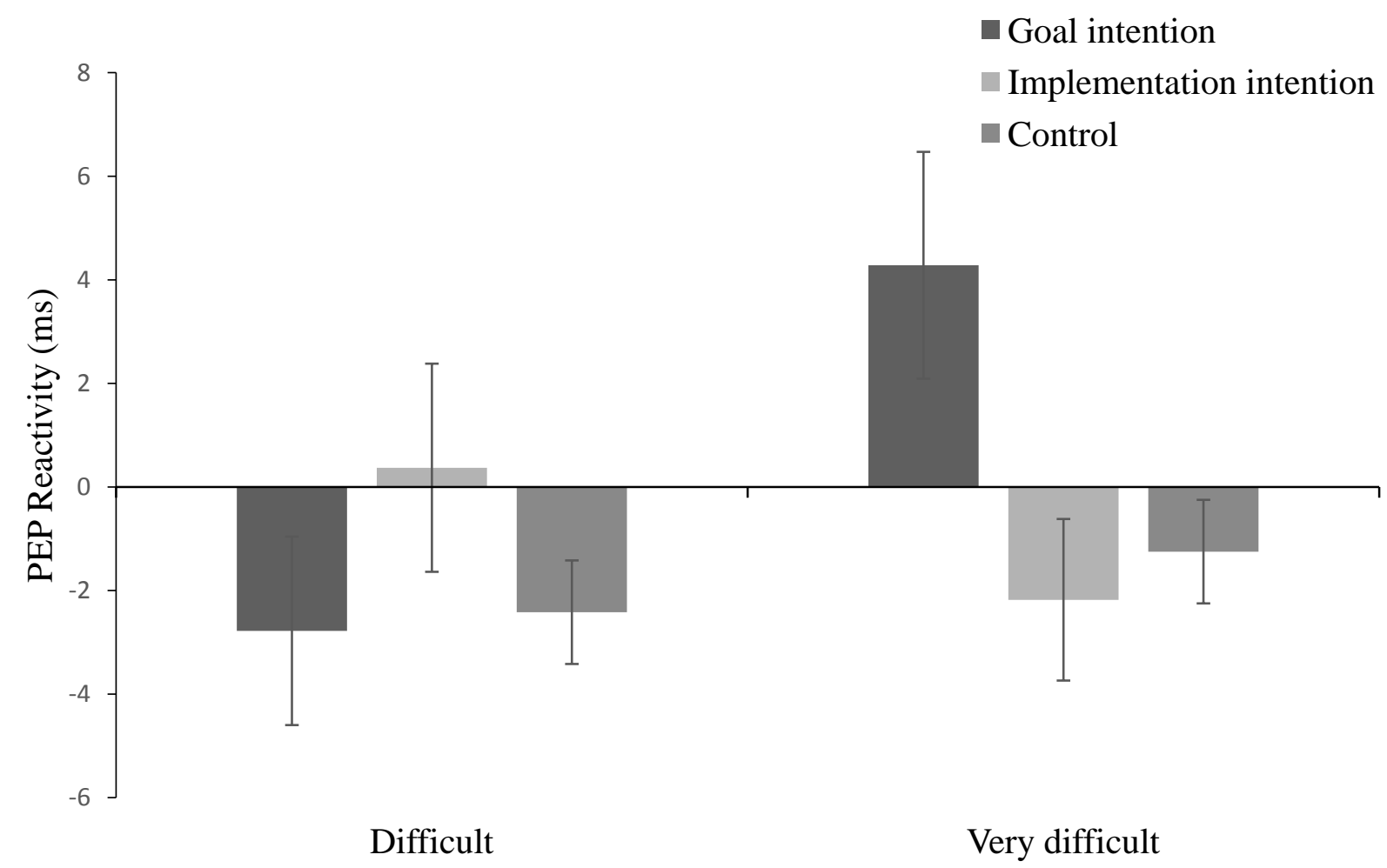


Figure 3

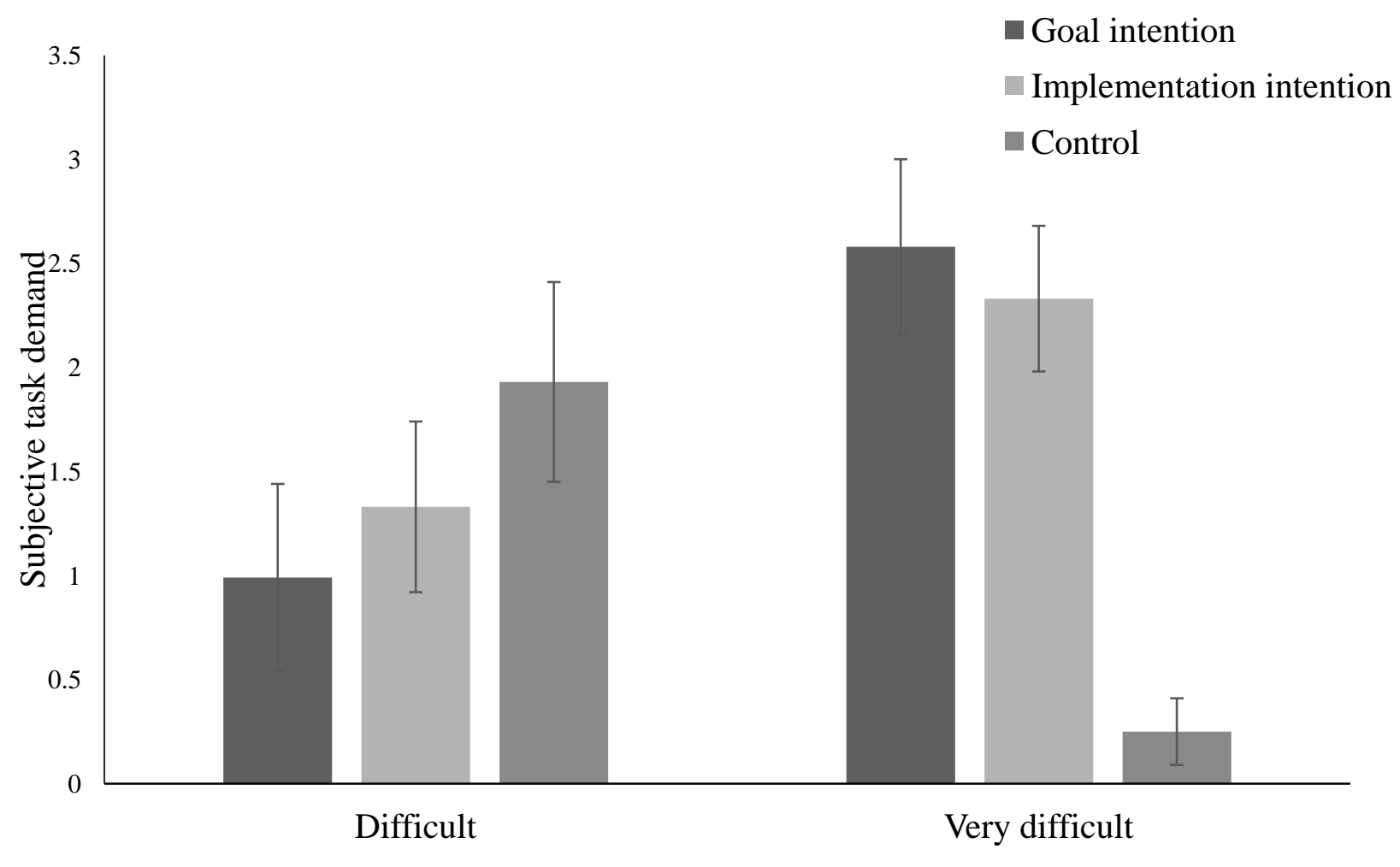

Task difficulty 
Table 1

Means (and Standard Errors) of the Cardiovascular Baseline Values in Experiment 1.

\begin{tabular}{cccc}
\hline & Goal intention & $\begin{array}{c}\text { Implementation } \\
\text { intention } \\
(n=16)\end{array}$ & Control \\
Cardiac PEP & $109.73(5.52)$ & $109.73(5.52)$ & $110.98(11.09)$ \\
SBP & $106.42(2.53)$ & $105.11(2.40)$ & $105.50(2.72)$ \\
DBP & $60.55(1.44)$ & $59.22(2.03)$ & $61.31(1.22)$ \\
HR & & & \\
& & & \\
& & & \\
\end{tabular}

Note. Cardiac $\mathrm{PEP}=$ pre-ejection period $($ in $\mathrm{ms}), \mathrm{SBP}=$ systolic blood pressure $($ in $\mathrm{mmHg})$, $\mathrm{DBP}=$ diastolic blood pressure (in $\mathrm{mmHg}), \mathrm{HR}=$ heart rate (beats/min). 
Table 2

Means (and Standard Errors) of the SPB, DBP and HR Reactivity Scores during Task Performance in Experiment 1.

\begin{tabular}{cccc}
\hline & Goal intention & $\begin{array}{c}\text { Implementation } \\
\text { intention } \\
(n=16)\end{array}$ & Control \\
SBP & $0.87(1.34)$ & $0.78(0.60)$ & $0.79(0.87)$ \\
DBP & $0.62(0.99)$ & $1.03(0.77)$ & $1.39(0.81)$ \\
HR & $-0.52(1.84)$ & $1.19(0.95)$ & $1.56(0.94)$ \\
\hline
\end{tabular}

Note. $\mathrm{SBP}$ and $\mathrm{DBP}=$ systolic and diastolic blood pressure (in $\mathrm{mmHg}$ ), $\mathrm{HR}=$ heart rate (beats/min). 
Table 3

Means (and Standard Errors) of the Cardiovascular Baseline Values in Experiment 2.

\begin{tabular}{ccccccc}
\hline \multicolumn{3}{c}{ Difficult task } \\
& $\begin{array}{c}\text { Goal } \\
\text { intention }\end{array}$ & $\begin{array}{c}\text { Implementa- } \\
\text { tion } \\
\text { intention } \\
(n=10)\end{array}$ & Control & $\begin{array}{c}\text { Goal } \\
\text { intention }\end{array}$ & $\begin{array}{c}\text { Implementa- } \\
\text { tion } \\
\text { intention } \\
(n=12)\end{array}$ & Control \\
& $(n=12)$ & $(n=15)$ & $(n=15)$ & $108)$ \\
\hline Cardiac & 114.57 & 116.75 & 121.87 & 113.08 & 108.78 & 110.37 \\
PEP & $(4.75)$ & $(5.02)$ & $(3.59)$ & $(6.26)$ & $(5.36)$ & $(6.91)$ \\
SBP & 110.07 & 109.78 & 108.47 & 106.45 & 107.73 & 107.54 \\
& $(2.63)$ & $(3.71)$ & $(2.80)$ & $(2.81)$ & $(2.13)$ & $(2.99)$ \\
DBP & 60.07 & 59.81 & 63.44 & 63.82 & 62.02 & 61.12 \\
& $(1.65)$ & $(1.79)$ & $(1.45)$ & $(1.80)$ & $(1.30)$ & $(1.93)$ \\
HR & 75.73 & 81.78 & 77.42 & 79.91 & 75.60 & 77.62 \\
& $(4.05)$ & $(4.13)$ & $(3.72)$ & $(4.08)$ & $(2.77)$ & $(3.63)$ \\
\hline
\end{tabular}

Note. Cardiac $\mathrm{PEP}=$ pre-ejection period (in $\mathrm{ms}), \mathrm{SBP}=$ systolic blood pressure (in $\mathrm{mmHg}$ ), $\mathrm{DBP}=$ diastolic blood pressure (in $\mathrm{mmHg}), \mathrm{HR}=$ heart rate (beats $/ \mathrm{min}$ ). 
Table 4

Means (and Standard Errors) of the SPB, DBP and HR Reactivity Scores during Task

Performance in Experiment 2.

\begin{tabular}{|c|c|c|c|c|c|c|}
\hline \multirow[t]{2}{*}{$\mathrm{c}$} & \multicolumn{3}{|c|}{ Difficult task } & \multicolumn{3}{|c|}{ Very difficult task } \\
\hline & $\begin{array}{c}\text { Goal } \\
\text { intention } \\
(n=12)\end{array}$ & $\begin{array}{c}\text { Implementa } \\
\text {-tion } \\
\text { intention } \\
(n=10)\end{array}$ & $\begin{array}{l}\text { Control } \\
(n=15)\end{array}$ & $\begin{array}{c}\text { Goal } \\
\text { intention } \\
(n=15)\end{array}$ & $\begin{array}{c}\text { Implementa } \\
\text {-tion } \\
\text { intention } \\
(n=12)\end{array}$ & $\begin{array}{l}\text { Control } \\
(n=8)\end{array}$ \\
\hline SBP & $\begin{array}{c}3.48 \\
(1.39)\end{array}$ & $\begin{array}{c}0.63 \\
(1.28)\end{array}$ & $\begin{array}{c}0.09 \\
(1.90)\end{array}$ & $\begin{array}{c}1.22 \\
(2.04)\end{array}$ & $\begin{array}{c}0.08 \\
(1.50)\end{array}$ & $\begin{array}{c}3.29 \\
(2.25)\end{array}$ \\
\hline DBP & $\begin{array}{c}1.15 \\
(1.09)\end{array}$ & $\begin{array}{c}1.82 \\
(1.39)\end{array}$ & $\begin{array}{l}-1.15 \\
(0.95)\end{array}$ & $\begin{array}{l}-1.97 \\
(0.53)\end{array}$ & $\begin{array}{c}0.25 \\
(1.28)\end{array}$ & $\begin{array}{l}-0.03 \\
(1.40)\end{array}$ \\
\hline HR & $\begin{array}{c}2.38 \\
(0.83)\end{array}$ & $\begin{array}{l}-0.01 \\
(0.92)\end{array}$ & $\begin{array}{c}0.83 \\
(0.73)\end{array}$ & $\begin{array}{c}0.70 \\
(0.90)\end{array}$ & $\begin{array}{c}2.78 \\
(1.02)\end{array}$ & $\begin{array}{l}-3.27 \\
(4.58)\end{array}$ \\
\hline
\end{tabular}

Note. $\mathrm{SBP}=$ systolic blood pressure (in $\mathrm{mmHg}$ ), $\mathrm{DBP}=$ diastolic blood pressure (in $\mathrm{mmHg}$ ), $\mathrm{HR}=$ heart rate (beats/min). 